\title{
RATING LONG-TERM USE OF SIMPLIFICATIONS IN TILLAGE AND PREVIOUS CROP ON BIOMETRIC FEATURES, PHYSIOLOGICAL AND YIELD OF WINTER WHEAT CULTIVAR 'Kobra Plus'
}

\section{OCENA WIELOLETNIEGO STOSOWANIA UPROSZCZEŃ W UPRAWIE ROLI ORAZ PRZEDPLONU NA CECHY BIOMETRYCZNE, FIZJOLOGICZNE I PLONOWANIE PSZENICY OZIMEJ ODMIANY 'Kobra Plus'}

\author{
Department of Agronomy, West Pomeranian University of Technology, Szczecin, Poland \\ ${ }^{1}$ Department of Meteorology and Green Areas Management, West Pomeranian University \\ of Technology, Szczecin, Poland
}

\begin{abstract}
Streszczenie. Badania przeprowadzono w latach 2006-2008 w doświadczeniu statycznym, założonym w 1993 roku, w Rolniczej Stacji Doświadczalnej Lipnik (k. Stargardu Szczecińskiego). Stacja jest położona w centralnej części Niziny Szczecińskiej ( $\varphi 5^{0} 21^{`} \mathrm{~N}, \lambda 1^{0} 58^{`} \mathrm{E}$, Hs 30 m n.p.m.). Badania polowe przeprowadzono na pszenicy ozimej uprawianej w zmianowaniu czteropolowym (burak cukrowy - pszenica ozima - bobik - pszenica ozima + międzyplon gorczyca biała). Badano wpływ stosowania uproszczeń w uprawie roli, polegających na eliminacji orki na rzecz uprawy bezpłużnej i siewu bezpośredniego, w odniesieniu do uprawy tradycyjnej, pod rośliny uprawiane w zmianowaniu. Badanym czynnikiem były systemy uprawy roli stosowane pod pszenicę ozimą po różnych przedplonach (bobik, burak cukrowy). Systemy uprawy istotnie kształtowały długość źdźbła i wskaźnik powierzchni asymilacyjnej liści (LAI). Pszenica ozima uprawiana na stanowisku po bobiku osiągała istotnie większe wartości krzewistości ogólnej i produkcyjnej, długości źdźbła i kłosa oraz wskaźnika zazielenienia liści (SPAD). Nie stwierdzono wpływu czynników doświadczalnych oraz ich współdziałania na plon ziarna pszenicy ozimej. Niezależnie od testowanych czynników doświadczenia pszenica ozima plonowała na poziomie $5 \mathrm{t} \cdot \mathrm{ha}^{-1}$. Nieznaczne tendencje do większego plonowania odnotowano w systemie tradycyjnym płużnym.
\end{abstract}

Key words: tillage systems, forecrop, winter wheat, biometric and physiological yield components.

Słowa kluczowe: systemy uprawy roli, przedplon, pszenica ozima, biometryczne i fizjologiczne komponenty plonu.

\section{INTRODUCTION}

Most of the field experiments, both worldwide and in Poland, upon the response of crops to reduction in the intensity of cultivation, were carried out using cereal plants, because their production profitability has an impact on the economy (Krzymuski 1998; Stolarski 2004). Although tillage simplifications reduce the energy inputs (Kostucic et al. 2001; Olson and

Corresponding author - Adres do korespodencji: Cezary Podsiadło, Department of Agronomy, West Pomeranian University of Technology, Szczecin, Juliusza Słowackiego 17, 71-434 Szczecin, Poland, e-mail: Cezary.Podsiadlo@zut.edu.pl 
Senjem 2002; Baker et al. 2007; Derpsch and Friedrich 2009) and associated costs, however, this may cause a decrease in yield (Dzienia et al. 2001; Lepiarczyk et al. 2006; Sekutowski 2007; Rieger et al. 2008). One of the main causes reducing the yield of cereals, by simplified tillage systems is to reduce the number of ears per $1 \mathrm{~m}^{2}$ (Małecka et al. 2012).

According to other authors, this effect should be combined with the occurrence of adverse weather course, because under favorable conditions, these reductions are small or do not exist (Anken et al. 2004; Golik et al. 2005).

There are studies that found an increase in the yield of winter wheat in the application of simplified tillage systems (Blecharczyk et al. 2006; De Vita et al. 2007).

In addition, the soil cultivated by the simplified scheme indicates a greater mass of plant residues than in the cultivated soil using plow.

Left in the soil, plant residues increase the stability of the agricultural ecosystems by the renewal of soil and maintain the positive balance of organic matter and its biological activity (Pałys et al. 2004).

Selection of an appropriate cultivation system is closely linked to the field crop production of a farm and soil conditions (Olson and Senjem 2002).

Location of Poland, and especially its north-west area, at the interface between maritime and continental climate, significantly determines the formation of pluvio-thermal conditions. Very frequent periods of rainfall shortage in spring and summer months are particularly dangerous. This situation, coupled with the predominant area of light soils, means that crops have unfavorable conditions for growth and development, the symptoms of which can be reduced dynamics of aboveground parts and roots growth, as well as deterioration in the yield structure components determining the size and quality of crops.

Use in these conditions minimized cultivation, reduces water and wind erosion, stimulates soil biodiversity, increases the content of organic matter and macro-and micronutrients (Dzienia et al. 2001; Weber 2002; Rokosz and Podsiadło 2015).

The aim of the study was to determine the response of winter wheat of Kobra Plus cv., by means of assessing the dynamics of canopy growth and yielding on a long-term use of simplifications in the pre-sowing tillage, depending on the forecrop applied.

\section{MATERIAL AND METHODS}

The study was conducted in a static experiment founded in 1993 in the Agricultural Experimental Station Lipnik (near Stargard Szczeciński). Localization of the station in the central part of Szczecin Lowland ( $\varphi 53^{0} 21^{`} \mathrm{~N}, \lambda 1^{0} 58^{`} \mathrm{E}$, Hs 30 m.a.s.I.) makes the measurement results are representative for the whole region, and even for western part of West Pomerania (Koźmiński and Michalska 2000). The field tests were carried out using winter wheat grown in four-field crop rotation (sugar beet - winter wheat - faba beans - winter wheat + white mustard intercrop). The experiment was founded on light soil of good rye complex and IVb bonitation class. The soil was developed from light loamy sand $(p / g)$, with weak loamy sand underneath ( $p s g)$, and light silt in some spots $(g /)$. In typological terms, this soil is counted to brown soils. Thickness of the humus layer is $14-25 \mathrm{~cm}$, humus content amounts to $1.3-1.5 \%$. Content of alluvial parts $11-13 \%, \mathrm{pH}_{\mathrm{KCL}} 6.0$, average content of $\mathrm{K}-105 \mathrm{mg} \cdot \mathrm{kg}^{-1}$ soil, $\mathrm{P}-79 \mathrm{mg} \cdot \mathrm{kg}^{-1}$ soil. The soil in terms of categories of agronomic soil is light typical for the region of Western Pomerania. 
Bi-factorial field experiment was established in the perpendicular bands pattern of randomized sub-blocks in four replicates. The studied factor consisted of tillage systems used for winter wheat cultivation after different forecrops (faba beans, sugar beet). Surface of the plot for harvest was $30 \mathrm{~m}^{2}$.

The first-order factor (tillage system): A - plowing tillage - classic tillage used for winter crops, $\mathrm{B}$ - no-plowing tillage - cultivator + string roller, $\mathrm{C}$ - direct sowing - sowing machine for direct sowing.

The second-order factor (forecrop): 1 - faba bean (self-completing 'Martin' cv.), 2 - sugar beet ('Kutnowska' cv.).

The winter wheat of 'Kobra Plus' cv. was sown in the experiment within the period recommended by agrotechnical procedures for Western Pomerania. For objects with conventional $(A)$ and plowless tillage $(B)$, wheat was sown using row cereal seeder. In contrast, on objects with direct sowing, a special seeder (for direct sowing) was applied. The frost-resistance of a variety is estimated for $4^{\circ}$ (in $9^{\circ}$ scale). It is resistant to lodging, sprouting, and shedding. It exhibits resistance to wheat stem rust and brown rust, and is also resistant to the take-all diseases.

Plant density [pcs $\cdot \mathrm{m}^{-2}$ ] - in autumn after emergence after, the framing by 2 times in each plot, with $0.5 \mathrm{~m}^{2}$, tota number of blades and, with ears - at full ripeness phase by means of square-frame method, twice from $0.5 \mathrm{~m}^{2}$ area, before winter wheat harvest - determination of the stalk length $[\mathrm{cm}]$ and spike length $[\mathrm{cm}]$ - for 30 random plants in each plot. Moreover, the coefficient LAI, chlorophyll content in the leaves in the shooting phase. The yield of winter wheat achieved from $30 \mathrm{~m}^{2}$ area recalculated onto $\mathrm{t} \cdot \mathrm{ha}^{-1}$. In all the years of research applied before sowing wheat, fertilization with nitrogen, phosphorus and potassium, respectively, in quantities of 24,80 , and $120 \mathrm{~kg} \cdot \mathrm{ha}^{-1}$. In the spring after the start of vegetation used only nitrogen fertilization, dividing them into two doses. Plant protection treatments were applied in accordance with the recommendations of agricultural technology used in the cultivation of winter wheat.

The study results were statistically processes using variance analysis for multi-year experiments, and difference significance at the level of $\alpha-0.05$, was evaluated applying Tukey's test in FR-ANALWAR 4.3. software based on Microsoft Excel (Rudnicki 2011).

Table 1 shows the average monthly temperatures and their deviation (in ${ }^{\circ} \mathrm{C}$ ) from the multi-year average of 1961 to 2000 and the classification of the thermal months Lorenc (2000) based on a standard deviation. Precipitation is characterized by the scale developed by Kaczorowska (1962), taking into account the percentage of rainfall in a given month in relation to the value of multi-year.

Among the analyzed period of years, where the average monthly air temperature considerably exceeded the standard began in June 2006 and lasted until mid - 2007 hottest and at the same time extremely dry was July 2006., The temperature of the month was nearly $6^{\circ} \mathrm{C}$ higher than the average, at very low rainfall $(10 \mathrm{~mm})$. Deficiencies rainfall persisted since the autumn of 2005 , which further worsened the conditions of the growing season, rainfall in 2006 accounted for less than $80 \%$ of normal, so this year was recognized as dry. In 2007 , the air temperature was much higher than average, except for the autumn months (IX-XI), but rain in most parts of the year were high, and the months from May to August, even extremely wet. The year 2008 was warmer than normal, and while annual rainfall was close to the average, their distribution was uneven, the worst moisture conditions prevailed in May and June. 
Table 1. Average monthly air temperature and precipitation in 2005-2008, together with a deviation from the norm (1961-2000) at the agrometeorological station in Lipnik

Tabela 1. Srednia miesięczna temperatura powietrza i sumy opadów atmosferycznych w latach 2005-2008, wraz z odchyleniem od normy (1961-2000) w stacji agrometeorologicznej w Lipniku

\begin{tabular}{|c|c|c|c|c|c|c|c|c|c|c|c|c|c|c|}
\hline & $\begin{array}{l}\text { Year/Deviation from the norm } \\
\text { Rok/Odchylenie od normy }\end{array}$ & I & II & III & IV & V & VI & VII & VIII & IX & $x$ & XI & XII & I-XII \\
\hline \multirow{9}{*}{ 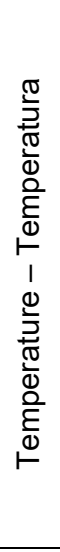 } & 2005 & & & - & - & - & - & - & - & 15.6 & 10.2 & 4.2 & 1.2 & \\
\hline & $\begin{array}{l}\text { deviation } \\
\text { odchylenie }\left[{ }^{\circ} \mathrm{C}\right]\end{array}$ & & & & & & & & & $\begin{array}{l}2.3 \\
b c\end{array}$ & $\begin{array}{c}1.4 \\
\mathrm{c}\end{array}$ & $\begin{array}{c}0.4 \\
\mathrm{n}\end{array}$ & $\begin{array}{c}0.8 \\
n\end{array}$ & \\
\hline & 2006 & -5.5 & -0.6 & 0.3 & 8.4 & 13.7 & 18.2 & 23.5 & 17.7 & 17.1 & 11.7 & 7.2 & 5.8 & 9.8 \\
\hline & $\begin{array}{l}\text { deviation } \\
\text { odchylenie }\left[{ }^{\circ} \mathrm{C}\right]\end{array}$ & $\begin{array}{l}-4.4 \\
\mathrm{ch}\end{array}$ & $\begin{array}{c}-0.3 \\
n\end{array}$ & $\begin{array}{l}-2.5 \\
\mathrm{ch}\end{array}$ & $\begin{array}{l}1.0 \\
\mathrm{Ic}\end{array}$ & $\begin{array}{l}1.0 \\
\mathrm{IC}\end{array}$ & $\begin{array}{l}2.2 \\
\mathrm{bc}\end{array}$ & $\begin{array}{l}5.9 \\
\text { ec }\end{array}$ & $\begin{array}{l}0.5 \\
\mathrm{n}\end{array}$ & $\begin{array}{l}3.8 \\
\text { ac }\end{array}$ & $\begin{array}{l}2.9 \\
\text { ac }\end{array}$ & $\begin{array}{l}3.4 \\
\mathrm{bc}\end{array}$ & $\begin{array}{l}5.4 \\
\mathrm{ac}\end{array}$ & $\begin{array}{l}1.6 \\
b c\end{array}$ \\
\hline & 2007 & 4.9 & 1.5 & 6.7 & 9.9 & 14.9 & 18.3 & 18.4 & 18.6 & 13.3 & 7.7 & 3.4 & 2.0 & 10.4 \\
\hline & $\begin{array}{l}\text { deviation } \\
\text { odchylenie }\left[{ }^{\circ} \mathrm{C}\right]\end{array}$ & $\begin{array}{l}6.0 \\
b c\end{array}$ & $\begin{array}{l}1.8 \\
\text { Ic }\end{array}$ & $\begin{array}{l}3.9 \\
b c\end{array}$ & $\begin{array}{l}2.5 \\
b c\end{array}$ & $\begin{array}{l}2.2 \\
b c\end{array}$ & $\begin{array}{l}2.3 \\
b c\end{array}$ & $\begin{array}{l}0.8 \\
\text { Ic }\end{array}$ & $\begin{array}{c}1.4 \\
\mathrm{C}\end{array}$ & $\begin{array}{l}0.0 \\
\mathrm{n}\end{array}$ & $\begin{array}{c}-1.1 \\
\text { Ich }\end{array}$ & $\begin{array}{c}-0.4 \\
\mathrm{n}\end{array}$ & $\begin{array}{l}1.6 \\
\text { Ic }\end{array}$ & $\begin{array}{l}2.2 \\
\text { ac }\end{array}$ \\
\hline & 2008 & 2.5 & 4.3 & 4.1 & 8.0 & 14.3 & 17.9 & 19.4 & 18.7 & 13.1 & 9.1 & 5.3 & 2.0 & 9.9 \\
\hline & $\begin{array}{l}\text { deviation } \\
\text { odchylenie }\left[{ }^{\circ} \mathrm{C}\right]\end{array}$ & $\begin{array}{l}3.6 \\
c\end{array}$ & $\begin{array}{l}4.6 \\
\mathrm{C}\end{array}$ & $\begin{array}{l}1.3 \\
\text { lc }\end{array}$ & $\begin{array}{c}0.6 \\
n\end{array}$ & $\begin{array}{l}1.6 \\
\text { lc }\end{array}$ & $\begin{array}{l}1.9 \\
b c\end{array}$ & $\begin{array}{l}1.8 \\
\mathrm{C}\end{array}$ & $\begin{array}{l}1.5 \\
\mathrm{C}\end{array}$ & $\begin{array}{c}-0.2 \\
\mathrm{n}\end{array}$ & $\begin{array}{c}0.3 \\
\mathrm{n}\end{array}$ & $\begin{array}{l}1.5 \\
\mathrm{lc}\end{array}$ & $\begin{array}{l}1.6 \\
\text { lc }\end{array}$ & $\begin{array}{l}1.7 \\
\mathrm{bc}\end{array}$ \\
\hline & $1961-2000$ & -1.1 & -0.3 & 2.8 & 7.4 & 12.7 & 16.0 & 17.6 & 17.2 & 13.3 & 8.8 & 3.8 & 0.4 & 8.2 \\
\hline \multirow{11}{*}{ 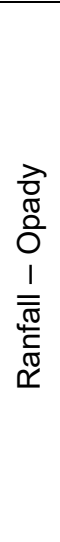 } & 2005 & - & - & - & - & - & - & - & - & 26 & 20 & 19 & 72 & \\
\hline & $\begin{array}{l}\text { the percentage norm } \\
\text { procent normy [\%] }\end{array}$ & & & & & & & & & $\begin{array}{r}55 \\
s\end{array}$ & $\begin{array}{r}51 \\
s\end{array}$ & $\begin{array}{l}46 \\
\text { bs }\end{array}$ & $\begin{array}{c}176 \\
\text { sw }\end{array}$ & \\
\hline & 2006 & 12 & 32 & 33 & 22 & 43 & 23 & 7 & 104 & 38 & 25 & 61 & 27 & 427 \\
\hline & the percentage norm & 34 & 123 & 97 & 58 & 83 & 37 & 10 & 192 & 81 & 64 & 149 & 66 & 79 \\
\hline & procent normy [\%] & bs & $\mathrm{n}$ & $\mathrm{n}$ & $s$ & $\mathrm{n}$ & bs & ss & sw & $\mathrm{n}$ & $s$ & w & $s$ & $s$ \\
\hline & 2007 & 76 & 42 & 54 & 4 & 105 & 109 & 109 & 103 & 47 & 14 & 55 & 34 & 752 \\
\hline & $\begin{array}{l}\text { the percentage norm } \\
\text { procent normy [\%] }\end{array}$ & $\begin{array}{r}217 \\
\text { sw }\end{array}$ & $\begin{array}{r}161 \\
\text { bw }\end{array}$ & $\begin{array}{r}159 \\
\text { bw }\end{array}$ & $\begin{array}{c}11 \\
\text { ss }\end{array}$ & $\begin{array}{r}202 \\
\text { sw }\end{array}$ & $\begin{array}{r}176 \\
\text { sw }\end{array}$ & $\begin{array}{c}163 \\
\text { bw }\end{array}$ & $\begin{array}{r}191 \\
\text { sw }\end{array}$ & $\begin{array}{r}100 \\
n\end{array}$ & $\begin{array}{l}36 \\
\text { bs }\end{array}$ & $\begin{array}{c}134 \\
w\end{array}$ & $\begin{array}{r}83 \\
n\end{array}$ & $\begin{array}{l}140 \\
\text { bw }\end{array}$ \\
\hline & 2008 & 59 & 23 & 46 & 109 & 10 & 30 & 35 & 49 & 47 & 68 & 36 & 40 & 552 \\
\hline & the percentage norm & 168 & 88 & 135 & 287 & 19 & 48 & 52 & 91 & 100 & 174 & 88 & 97 & 103 \\
\hline & procent normy [\%] & bw & $\mathrm{n}$ & w & sw & ss & bs & $\mathrm{s}$ & $\mathrm{n}$ & $\mathrm{n}$ & bw & $\mathrm{n}$ & $\mathrm{n}$ & $\mathrm{n}$ \\
\hline & $1961-2000$ & 35 & 26 & 34 & 38 & 52 & 62 & 67 & 54 & 47 & 39 & 41 & 41 & 536 \\
\hline
\end{tabular}

Thermal classification months - Termiczna klasyfikacja miesięcy: ec - extremely warm - ekstremalnie ciepły, ac - anomalously warm - anomalnie ciepły, bc - very warm bardzo ciepły, c - warm - ciepły, lc - slightly warm - lekko ciepły, $\mathrm{n}$ - normal - normalny, Ich - slightly cool - lekko chłodny , ch - cool - chłodny.

Drop classification months - Opadowa klasyfikacja miesięcy: ss - extremely dry - skrajnie suchy, bs - very dry - bardzo suchy, $\mathrm{s}$ - dry - suchy, $\mathrm{n}$ - normal - normalny,

w - wet - wilgotny, bw - very wet - bardzo wilgotny, sw - extreme wet - skrajnie wilgotny. 


\section{RESULTS AND DISCUSSION}

Based on studies conducted in 2006-2008, the impact of tillage systems and forecrop on biometric properties of winter wheat was statistically demonstrated (Table 2).

Table 2. The significance of main effects and interactions to impact system (S) tillage and previous crop $(\mathrm{P})$ on the properties of the selected biometric and physiological characteristics of winter wheat variety 'Kobra Plus' 2006-2008 (L)

Tabela 2. Istotność efektów głównych oraz interakcji dla wpływu systemu (S) uprawy oraz przedplonu (P) na właściwości biometryczne oraz wybrane cechy fizjologiczne pszenicy ozimej odmiany 'Kobra Plus' w latach 2006-2008 (L)

\begin{tabular}{|c|c|c|c|c|c|c|c|c|c|}
\hline \multirow[b]{2}{*}{ 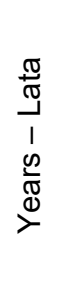 } & \multirow[b]{2}{*}{$\begin{array}{l}\text { Types of } \\
\text { variation } \\
\text { Rodzaje } \\
\text { zmienności }\end{array}$} & \multicolumn{8}{|c|}{ Characteristic - Cecha } \\
\hline & & $\begin{array}{c}\text { yield } \\
\text { plon } \\
{\left[\mathrm{t} \cdot \mathrm{ha}^{-1}\right]}\end{array}$ & $\begin{array}{c}\text { number } \\
\text { of plants } \\
\text { obsada } \\
\text { roślin } \\
{\left[\mathrm{m}^{-2}\right]}\end{array}$ & $\begin{array}{l}\text { total number } \\
\text { of blades } \\
\text { liczba źdźbeł } \\
\text { ogółłem } \\
{\left[\mathrm{m}^{-2}\right]}\end{array}$ & $\begin{array}{l}\text { the number of } \\
\text { blades, with } \\
\text { ears } \\
\text { liczba źdźbeł } \\
\text { z kłosami } \\
{\left[\mathrm{m}^{-2}\right]}\end{array}$ & $\begin{array}{c}\text { steam } \\
\text { length } \\
\text { długość } \\
\text { źdźbła } \\
{[\mathrm{cm}]}\end{array}$ & $\begin{array}{c}\text { ear } \\
\text { length } \\
\text { długość } \\
\text { kłosa } \\
{[\mathrm{cm}]}\end{array}$ & LAI & SPAD \\
\hline \multirow{3}{*}{ ஜ̊ } & $S$ & * & - & * & - & * & - & - & - \\
\hline & $P$ & - & - & - & * & * & - & - & * \\
\hline & $S \cdot P$ & - & * & - & - & - & * & - & * \\
\hline \multirow{3}{*}{ 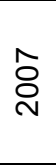 } & $S$ & - & - & - & - & - & * & * & - \\
\hline & $P$ & * & - & - & - & - & - & - & * \\
\hline & $S \cdot P$ & - & - & * & - & - & * & - & - \\
\hline \multirow{3}{*}{$\stackrel{\infty}{\circ}$} & $S$ & - & * & - & - & - & * & - & - \\
\hline & $P$ & - & - & - & * & - & * & - & - \\
\hline & $S \cdot P$ & - & - & - & - & - & - & - & - \\
\hline \multirow{6}{*}{$\begin{array}{l}\infty \\
\stackrel{े}{1} \\
\text { 1 } \\
\varnothing \\
\stackrel{N}{0}\end{array}$} & S & - & - & - & - & * & - & * & - \\
\hline & $L \cdot S$ & - & - & - & - & * & * & - & - \\
\hline & $P$ & - & - & - & * & * & * & - & * \\
\hline & $L \cdot P$ & - & - & - & - & - & - & - & - \\
\hline & $S \cdot P$ & - & - & * & * & - & - & - & - \\
\hline & $L \cdot S \cdot P$ & - & - & - & - & * & * & - & - \\
\hline
\end{tabular}

* a significant effect - efekt istotny, „-" - not significant effect - efekt nieistotny.

A study conducted in 1996-1998 in ZDD Brody showed that winter wheat grain yield applying conventional tillage was higher by $0.72 \mathrm{t} \cdot \mathrm{ha}^{-1}$ as compared to the yield obtained due to the direct sowing. The difference in favor of the traditional cultivation was underlined in 1996 and 1997, while in the last year of the study there was no difference in the level of yields between tillage systems. Wheat grown in direct sowing was characterized by a smaller spike density (Blecharczyk et al 1999).

Jędruszczak and Antoszek (2004) claimed that the highest grain yields were obtained from plowing cultivation, and any deviation from this system was accompanied by a reduction in grain yield. Replacement of the pre-sowing plowing with disking or cultivating resulted in the decrease of yield by $9 \%$ and $12 \%$, and the use of direct sowing resulted in a $17 \%$ yield reduction. 
The number of spikes is determined by the productive tillering, which largely depends on the environmental conditions (Mittler 2000).

Among the biometric features in our study, cultivation systems differentiated only the length of stalks, which was the largest in the traditional tillage $(84.3 \mathrm{~cm})$ and the smallest in direct sowing $(80.80 \mathrm{~cm})$. The leaf assimilation index - LAl varied in a similar manner (Table 3 and 4).

Table 3.The influence of $(\mathrm{S})$ tillage system and previous crop (P) on the properties of biometric winter wheat varieties 'Kobra Plus'

Tabela 3. Wpływ systemu (S) uprawy oraz przedplonu (P) na właściwości biometryczne pszenicy ozimej odmiany 'Kobra Plus'

\begin{tabular}{|c|c|c|c|c|c|c|c|c|c|}
\hline \multirow{2}{*}{$\begin{array}{l}\text { Characteristic } \\
\text { Cecha }\end{array}$} & \multirow{2}{*}{$\begin{array}{l}\text { Years } \\
\text { Lata }\end{array}$} & \multicolumn{3}{|c|}{$\begin{array}{l}\text { Tillage system } \\
\text { System uprawy }\end{array}$} & \multicolumn{2}{|c|}{$\begin{array}{l}\text { Previous crop } \\
\text { Przedplon }\end{array}$} & \multirow{2}{*}{$\begin{array}{l}\text { Mean } \\
\text { Średnia }\end{array}$} & \multicolumn{2}{|c|}{$\begin{array}{l}\text { LSD }_{0.05} \text { for } \\
\text { NIR }_{0.05} \text { dla }\end{array}$} \\
\hline & & $A$ & $B$ & C & 1 & 2 & & $S$ & $P$ \\
\hline \multirow{4}{*}{$\begin{array}{l}\text { Number of } \\
\text { plants } \\
\text { Obsada roślin } \\
{\left[\mathrm{m}^{-2}\right]}\end{array}$} & 2006 & 500 & 490 & 485 & 595 & 494 & 513 & ns. & ns. \\
\hline & 2007 & 480 & 470 & 490 & 490 & 470 & 480 & ns. & ns. \\
\hline & 2008 & 490 & 480 & 500 & 500 & 480 & 490 & ns. & ns. \\
\hline & $\begin{array}{c}2006- \\
2008\end{array}$ & 490 & 480 & 495 & 495 & 480 & 488 & ns. & ns. \\
\hline \multirow{4}{*}{$\begin{array}{l}\text { Total number } \\
\text { of steam } \\
\text { Liczba źdźbeł } \\
\text { ogółem } \\
{\left[\mathrm{m}^{-2}\right]}\end{array}$} & 2006 & 640 & 560 & 580 & 660 & 530 & 594 & ns. & ns. \\
\hline & 2007 & 450 & 480 & 480 & 475 & 470 & 471 & ns. & 70.549 \\
\hline & 2008 & 650 & 570 & 560 & 655 & 525 & 592 & ns. & 32.663 \\
\hline & $\begin{array}{c}2006- \\
2008\end{array}$ & 570 & 550 & 570 & 560 & 520 & 550 & ns. & 33.525 \\
\hline \multirow{4}{*}{$\begin{array}{l}\text { The number of } \\
\text { steam with } \\
\text { ears } \\
\text { Liczba źdźbeł } \\
\text { z kłosami } \\
{\left[\mathrm{m}^{-2}\right]}\end{array}$} & 2006 & 632 & 557 & 557 & 646 & 518 & 582 & ns. & 80.697 \\
\hline & 2007 & 426 & 470 & 471 & 456 & 456 & 456 & ns. & ns. \\
\hline & 2008 & 632 & 557 & 557 & 646 & 518 & 582 & ns. & 75.541 \\
\hline & $\begin{array}{c}2006- \\
2008\end{array}$ & 564 & 528 & 528 & 583 & 497 & 540 & ns. & 37.664 \\
\hline \multirow{4}{*}{$\begin{array}{l}\text { Steam length } \\
\text { Długość } \\
\text { źdźbła } \\
{[\mathrm{cm}]}\end{array}$} & 2006 & 91.5 & 89.21 & 87.10 & 92.69 & 85.86 & 89.28 & 2.958 & 2.178 \\
\hline & 2007 & 77.5 & 78.22 & 75.91 & 76.82 & 77.51 & 77.17 & ns. & ns. \\
\hline & 2008 & 84 & 80.66 & 79.35 & 80.95 & 81.64 & 81.30 & ns. & ns. \\
\hline & $\begin{array}{c}2006- \\
2008\end{array}$ & 84.30 & 82.70 & 80.80 & 83.50 & 81.70 & 82.60 & 2.280 & 1.525 \\
\hline \multirow{4}{*}{$\begin{array}{l}\text { Ear length } \\
\text { Długość kłosa } \\
\text { [cm] }\end{array}$} & 2006 & 7.40 & 7.57 & 7.22 & 7.77 & 7.02 & 7.39 & ns. & ns. \\
\hline & 2007 & 8.38 & 7.79 & 7.94 & 8.00 & 8.08 & 8.04 & 0.276 & ns. \\
\hline & 2008 & 7.22 & 7.25 & 7.84 & 7.70 & 7.17 & 7.43 & 0.403 & 0.295 \\
\hline & $\begin{array}{c}2006- \\
2008\end{array}$ & 7.66 & 7.53 & 7.66 & 7.82 & 7.42 & 7.62 & ns. & 0.183 \\
\hline
\end{tabular}

A - ploughing - płużny, B - ploughless - bezpłużny, C - direct sowing - siew bezpośredni, 1 - faba bean - bobik, 2 - sugar beet - burak cukrowy, ns. - not significant - nieistotne.

In the case of the forecrop, diversity concerned: total number of steam and, with ears, length of stalks and spikes, as well as the ratio of leaf greenness - SPAD (Table 3, 4). All these features had higher values in the position after faba bean. The interaction of both experimental factors differentiated only total number of steam and, with ears. In our study, there was no the effect of experimental factors and their interaction on grain yield of winter wheat. 
Table 4. The influence of $(S)$ tillage system and previous crop $(P)$ on the properties of the selected biometric and physiological characteristics of winter wheat cultivar 'Kobra Plus'

Tabela 4. Wpływ systemu (S) uprawy oraz przedplonu $(P)$ na właściwości biometryczne oraz wybrane cechy fizjologiczne pszenicy ozimej odmiany 'Kobra Plus'

\begin{tabular}{|c|c|c|c|c|c|c|c|c|c|}
\hline \multirow{2}{*}{$\begin{array}{l}\text { Characteristic } \\
\text { Cecha }\end{array}$} & \multirow{2}{*}{$\begin{array}{l}\text { Years } \\
\text { Lata }\end{array}$} & \multicolumn{3}{|c|}{$\begin{array}{l}\text { Tillage system } \\
\text { System uprawy }\end{array}$} & \multicolumn{2}{|c|}{$\begin{array}{l}\text { Previous crop } \\
\text { Przedplon }\end{array}$} & \multirow{2}{*}{$\begin{array}{l}\text { Mean } \\
\text { Srednia }\end{array}$} & \multicolumn{2}{|c|}{$\begin{array}{l}\mathrm{LSD}_{0.05} \text { for } \\
\mathrm{NIR}_{0,05} \mathrm{dla}\end{array}$} \\
\hline & & $\mathrm{A}$ & $\mathrm{B}$ & $\mathrm{C}$ & 1 & 2 & & $S$ & $\mathrm{P}$ \\
\hline \multirow{4}{*}{$\begin{array}{l}\text { Grain field } \\
\text { Plon ziarna } \\
{\left[\mathrm{t} \cdot \mathrm{ha}^{-1}\right]}\end{array}$} & 2006 & 4.64 & 4.43 & 4.11 & 4.35 & 4.43 & 4.39 & \multirow{4}{*}{$\begin{array}{c}0.336 \\
\text { ns. } \\
\text { ns. } \\
\text { ns. }\end{array}$} & \multirow{4}{*}{$\begin{array}{l}\text { ns. } \\
\text { ns. } \\
\text { ns. } \\
\text { ns. }\end{array}$} \\
\hline & 2007 & 6.45 & 6.44 & 5.98 & 6.08 & 6.50 & 6.29 & & \\
\hline & 2008 & 3.94 & 4.39 & 4.63 & 4.32 & 4.32 & 4.32 & & \\
\hline & $\begin{array}{c}2006- \\
2008\end{array}$ & 5.00 & 5.09 & 4.91 & 4.92 & 5.08 & 5.00 & & \\
\hline \multirow{4}{*}{ LAI } & 2006 & 2.78 & 3.05 & 2.58 & 2.83 & 2.78 & 2.80 & \multirow{4}{*}{$\begin{array}{c}\text { ns. } \\
0.495 \\
\text { ns. } \\
0.355\end{array}$} & \multirow{4}{*}{$\begin{array}{l}\text { ns. } \\
\text { ns. } \\
\text { ns. } \\
\text { ns. }\end{array}$} \\
\hline & 2007 & 3.50 & 3.24 & 2.91 & 3.11 & 3.33 & 3.22 & & \\
\hline & 2008 & 3.95 & 3,35 & 3.45 & 3.61 & 3.56 & 3.58 & & \\
\hline & $\begin{array}{c}2006- \\
2008\end{array}$ & 3.40 & 3.20 & 3.00 & 3.20 & 3.20 & 3.20 & & \\
\hline \multirow{4}{*}{ SPAD } & 2006 & 47.92 & 48.31 & 47.98 & 51.36 & 44.77 & 48.07 & ns. & \multirow{4}{*}{$\begin{array}{c}1.264 \\
1.140 \\
\text { ns. } \\
1.115\end{array}$} \\
\hline & 2007 & 48.60 & 48.98 & 48.28 & 47.75 & 49.49 & 48.62 & \multirow{3}{*}{$\begin{array}{l}\text { ns. } \\
\text { ns. } \\
\text { ns. }\end{array}$} & \\
\hline & 2008 & 41.36 & 38.24 & 40.36 & 40.85 & 39.12 & 39.99 & & \\
\hline & $\begin{array}{c}2006- \\
2008\end{array}$ & 46.00 & 45.20 & 45.50 & 46.60 & 44.50 & 45.60 & & \\
\hline
\end{tabular}

Explanations as in Table 3 - Objaśnienia jak w tab. 3.

In the literature, opinions about impact on the biometric and physiological features of these experimental factors are very diverse. Significant effects of the tillage systems in relation to the length of the stalk, were pointed out by Dzienia and Wereszczaka (1999). Instead, like in own study, these authors along with Orzech et al. (2002), Weber and Hryńczuk (2004), as well as Kuś (1999) reported that cultivation systems have no significant influence on the length of spike and grain yield. The latter author also points to the presence of the trend of lower yielding in conventional tillage. Our findings do not confirm such result. On the contrary, the tendency to lower yields was observed in the direct sowing. Among authors confirming the significant effect on biometrics and plant yielding, following should be listed: Kuś (1999) as well as Orzech et al. (2002) - plant density, Dzienia and Dojss (1999), Weber and Hryńczuk (2004) - productive tillering, Włodek et al. (1999) - plowless tillage can cause lower plant density and in years with rainfall deficiency, also lower grain yields.

A number of authors indicate higher yielding of cereal crops in the traditional and simplified tillage systems, while the lowest in direct sowing (Dzienia and Dojss 1999; Dzienia and Wereszczaka 1999; Orzech et al. 2002).

Study of Biskupski et al. (2009) confirmed that, regardless of experimental factors examined, the leaf assimilation index (LAI), average angle of leaf inclination (MTA), and yield of selected spring wheat varieties, were affected exclusively by weather conditions. 


\section{CONCLUSIONS}

1. Tillage system significantly shaped the length of stalks and leaf assimilation index (LAl). Winter wheat grown in the position after faba bean had significantly higher values of total number of steam and, with ears, the length of stalk and spike, as well as the ratio of leaf greenness (SPAD). In our study, there was no the effect of experimental factors and their interaction on grain yield of winter wheat.

2. In the first year of study (12 year of experience) reported uneven growth and development of winter wheat depending on the agents tested experience. In subsequent years experience ontogenesis of winter wheat he was as balanced, with a slight tendency in favor of the classical plow cultivation.

3. Regardless of the experimental factors tested, winter wheat yielded at the level of $5 \mathrm{t} \cdot \mathrm{h}^{-1}$. Slight tendencies to a higher yielding was recorded at the traditional plowing system.

\section{REFERENCES}

Anken T., Weisskopf P., Zihlmann U., Forrer H., Jansa J., Perhacova K. 2004. Long-term tillage systems effects under moist cool conditions in Switzerland. Soil Till. Res. 78, 171-183.

Baker J.M., Ochsner T.E., Venterrea R.T., Griffis T.J. 2007. Tillage and soil carbon sequestration What do we realy know ? Agric. Ecosys. Environ. 118, 1-5.

Biskupski A., Włodek S., Pabin J. 2009. Wpływ zróżnicowanej uprawy roli na wybrane wskaźniki architektury łanu i plonowanie roślin [The influence of differentiated tillage on selected indices of canopy architecture and yield of plants]. Fragm. Agron. 26(4), 7-13. [in Polish]

Blecharczyk A., Skrzypczak G., Małecka I. 1999. Reakcja pszenicy ozimej na przedplon i siew bezpośredni [The reaction of winter wheat forecrop and direct sowing]. Pamięt. Puł. 118, 9-16. [in Polish]

Blecharczyk A., Śpitalniak J., Małecka I. 2006. Wpływ doboru przedplonów oraz systemów uprawy roli i nawożenia azotem na plonowanie pszenicy ozimej [The impact of selection previous cropping and tillage systems and nitrogen fertilization on yield of winter wheat]. Fragm. Agron. 23(2), 273-286. [in Polish]

De Vita P., Di Paolo E., Fecondo G., Di Fonzo N., Pisante M. 2007. No-tillage and conventional tillage effects on durum wheat yield, grain quality and soil moisture content in southern Italy. Soil Till. Res. 92, 69-78.

Derpsch R., Friedrich T. 2009. Global overview of conservation agriculture adoption. Proceed, in: 4th World Congress on Conservation Agriculture, New Delhi, India, 4-7 February 2009. [b.w.], 429-438.

Dzienia S., Dojss D. 1999. Wpływ systemów uprawy roli na zachwaszczenie i plonowanie pszenicy ozimej [Influence of tillage systems on weed infestation and yield of winter wheat]. Zesz. Nauk. AR Szczec., Agricultura 195(74), 185-190. [in Polish]

Dzienia S., Wereszczaka J. 1999. Efektywność systemów uprawy roli pod pszenicę ozimą na glebie kompleksu pszennego dobrego [The effectiveness of tillage systems in winter wheat on the soil of good wheat complex]. Zesz. Nauk. AR Szczec., Agricultura 195(74), 181-184. [in Polish]

Dzienia S., Pużyński S., Wereszczaka J. 2001. Impact of soil cultivation systems on chemical properties. Electron. J. Pol. Agric., Ser. Agronomy 4(2), www.ejpau.media.pl, access: December 2016. ISSN 1505-0297.

Golik S., Chidichimo H., Sarandon S. 2005. Biomass production, nitrogen accumulation and yield in wheat under two tillage systems and nitrogen supply in the Argentine Rolling Pampa. World J. Agric. Sci. 1(1), 36-41. 
Jędruszczak M., Antoszek R. 2004. Sposoby uprawy roli a bioróżnorodność zbiorowisk chwastów w monokulturze pszenicy ozimej [The methods of cultivation and the biodiversity of weeds in monoculture of winter wheat]. Acta Sci. Pol., Agricultura 3(2), 47-59. [in Polish]

Kaczorowska Z. 1962. Opady w Polsce w przebiegu wieloletnim [Precipitation in Poland in the course of many years]. Pr. Geograf. PAN 33, 107. [in Polish]

Kostucic S., Dubravko F., Gospodarić Z. 2001. Maize and Winter wheat production with different soil tillage systems on soil loam. Agric. Food Finland 10, 81-90.

Koźmiński C., Michalska B. 2000. Klimatyczna charakterystyka rejonu stacji agrometeorologicznej w Lipkach k. Stargardu Szczecińskiego. Szczecin, Wydaw. AR w Szczecinie, 10-30. ISBN 83-87327-53-0. [in Polish]

Krzymuski J. 1998. Zmiany w strukturze zasiewów i wartości przedplonów zbóż w latach 1971-1995 [Changes in crop structure and the previous cropping of cereals in the years 1971-1995]. Rocz. Nauk. Rol., Ser. A Prod. Rośl. 113(1-2), 9-20. [in Polish]

Kuś J. 1999. Wpływ różnej intensywności uprawy roli na jej właściwości i plonowanie roślin [The impact of varying the intensity of tillage on its properties and yield of plants]. Zesz. Nauk. AR Szczec., Agricultura 195(74), 33-38. [in Polish]

Lepiarczyk A. Kulig B., Stępnik K. 2006. Wpływ uproszczeń uprawy roli na plonowanie oraz kształtowanie się wskaźnika powierzchni liści jęczmienia jarego i bobku [The impact of simplification of tillage on yield and formation of leaf area index of spring barley and faba Bean]. Fragm. Agron. 2(90), 251-260. [in Polish]

Lorenc H. 2000. Termiczno-opadowa ocena klimatycznych sezonów roku w Polsce oraz tendencje czasowo-przestrzenne. Projekt badawczy M-9. Puławy, IMGW (typescript). [in Polish]

Małecka I., Blecharczyk A., Sawińska Z., Piechota T., Waniorek B. 2012. Plonowanie zbóż w zależności od sposobów uprawy roli [Yields of cereals depending on the different farming]. Fragm. Agron. 29(1), 114-123. [in Polish]

Mittler S. 2000. Ökovariabilität von Winterweizen unter Standortbedingungen Nordostdeutschlands (Winter wheat changeability under north Germany conditions). Disseration an der Landwirtschaftlich-Gärtnerischen, Fakultät der Humboldt Universität zu Berlin.

Olson K.D., Senjem N.B. 2002. Economic comparison of international changes in till systems in the Minesota River Basin Plan. Publ. Univ. of Minestota,1-118.

Orzech K., Nowicki J., Wanic M. 2002. Plonowanie pszenicy ozimej w zależności od sposobu uprawy gleby średniej [Yields of winter wheat depending on how the cultivation of the soil medium]. Pamięt. Puł. 130, 523-529. [in Polish]

Pałys E., Kraska P., Kuraszkiewicz R. 2004. Wpływ systemów uprawy roli na masę resztek pożniwnych pszenicy ozimej uprawianej na rędzinie [The impact of tillage systems on the weight of crop residues of winter wheat grown on rędzina]. Ann. UMCS, Sec. E 59(2), 527-533. [in Polish]

Rieger S., Richner W., Streit B., Frossard E., Liedgens M. 2008. Growth, yield, and yield components of winter wheat and the effects of tillage intensity, preceding crops, and $\mathrm{N}$ fertilisation. Eur. J. Agron. 28, 405-411.

Rokosz E., Podsiadło C. 2015. Wpływ deszczowania, system uprawy i nawożenia mineralnego na plonowanie i właściwości fizyczne gleby lekkiej w uprawie odmian bobiku [The impact of irrigation, tillage system and fertilization on yield and soil physical properties of light in the cultivation of varieties of faba bean]. Infrastrukt. Ekol. Ter. Wiej. 3(1), 625-636. [in Polish]

Rudnicki F. 2011. FR-ANALWAR 4.3. Software based on Microsoft Excel. Bydgoszcz, University of Science and Technology, Poland.

Stolarski T. 2004. Kierunki przemian w strukturze producentów zbóż [Directions of changes in the structure of grain producers]. Wieś Jutra 3(68), 10-12. [in Polish]

Sekutowski T. 2007. Wpływ technologii uprawy i ochrony herbicydowej na wysokość plonu pszenicy ozimej uprawianej w monokulturze [The impact of cultivation and protection of herbicidal the height of the yield of winter wheat cultivated in monoculture]. Inż. Rol. 3(91), 159-166. [in Polish] 
Weber R. 2002. Wpływ uprawy zachowawczej na ochronę środowiska [The impact of growing conservative on environmental protection]. Post. Nauk Rol. 1, 57-67. [in Polish]

Weber R., Hryńczuk B. 2004. Wpływ uproszczeń w uprawie roli na zmienność plonowania i komponentów plonu kilku odmian pszenicy ozimej [The impact of simplification in the cultivation of the role of the variability of yield and yield components of some varieties of winter wheat]. Ann. UMCS, Sec. E 59(1), 501-508. [in Polish]

Włodek S., Pabin J., Biskupski A., Kuś A. 1999. Skutki uproszczeń uprawy roli w zmianowaniu [The effects of simplification cultivation in crop rotation]. Zesz. Nauk. AR w Szczec., Agricultura 195(74), 39-45. [in Polish]

Abstract. The study was conducted in the years 2006-2008, in a static experiment founded in 1993 in the Agricultural Experimental Station Lipnik (near Stargard Szczeciński). The station is located in the central part of the Szczecin Lowland ( $\varphi 53^{0} 21^{`} \mathrm{~N}, \lambda 14^{0} 58^{\circ} \mathrm{E}$, Hs 30 m.a.s.I.). Field tests were carried out using winter wheat grown in four-field crop rotation (sugar beet - winter wheat - faba beans - winter wheat + white mustard intercrop). The effect of simplified tillage involving the elimination of plowing for the plowless tillage and direct sowing was examined, as compared to conventional tillage under crops grown in rotation. Studied factor consisted of the farming systems used for winter wheat after different forecrops (faba beans, sugar beet). The tillage systems significantly shaped the length of the stalk and leaf assimilation index (LAI). Winter wheat grown in the field after faba bean had significantly higher values of total number of steam and, with ears, the length of stalk and spike, as well as the ratio of leaf greenness (SPAD). In our study, there was no the effect of experimental factors and their interaction on grain yield of winter wheat. Regardless of tested experimental factors, winter wheat yielded at the level of $5 \mathrm{t} \cdot \mathrm{ha}^{-1}$. A slight tendency to higher yields was recorded for the traditional plowing system. 